\title{
Author Correction: Raynaud phenomenon and digital ulcers in systemic sclerosis
}

\author{
Michael Hughes (D), Yannick Allanore, Lorinda Chung, John D. Pauling (D), Christopher P. Denton and Marco Matucci-Cerinic
}

Correction to: Nature Reviews Rheumatology (2020) https://doi.org/10.1038/s41584-020-0386-4, published online 25 February 2020.

In the originally published version of this article there was an error in the text. The sentence "For example, if the ulcer is 'wet' then appropriate dressings that contain hydrogels and hydrocolloids should be selected with the aims of reducing moisture and drying the wound" has been corrected to "For example, if the ulcer is 'dry' then appropriate dressings that contain hydrogels and hydrocolloids should be selected with the aims of promoting moisture and avoiding drying the wound." This error has now been corrected in the HTML and PDF versions of the manuscript.

https://doi.org/10.1038/s41584-021-00591-5 I Published online 9 March 2021

(c) Springer Nature Limited 2021

\section{Author Correction: Location, location, location: how the tissue microenvironment affects inflammation in RA}

\author{
Christopher D. Buckley, Caroline Ospelt (1), Steffen Gay 10 and Kim S. Midwood (D)
}

Correction to: Nature Reviews Rheumatology (2021) https://doi.org/10.1038/s41584-020-00570-2, published online 01 February 2021.

In the originally published version of this article there was an error in Table 1. The phenotypes for immunomodulatory sub-lining fibroblasts and perivascular sub-lining fibroblasts were mistakenly swapped. In the row entitled "Sub-lining layer (immunomodulatory)", the cell for "Marker genes (human)" has been corrected from "Negative (CD34); positive (CD90 and HLA-DRA)" to "Positive (CD90 and CD34)" and the cell for "Marker genes (mouse)" has been corrected from "Negative (Cd34); positive (Cd90)" to "Positive (Cd90 and Cd34)". In the row entitled "Sub-lining layer (perivascular)", the cell for "Marker genes (human)" has been corrected from "Positive (CD90 and CD34)" to "Negative (CD34); positive (CD90 and HLA-DRA)" and the cell for "Marker genes (mouse)" has been corrected from "Positive (Cd90 and Cd34)" to "Negative (Cd34); positive (Cd90)". This error has now been corrected in the HTML and PDF versions of the manuscript.

https://doi.org/10.1038/s41584-021-00602-5 I Published online 12 March 2021

(C) Springer Nature Limited 2021 